\title{
Giant thymic cyst in left lower hemithorax of a healthy teenaged athlete
}

\author{
Cathrine Constantacos, MD, ${ }^{\text {a,e }}$ Nicholette M. Lawson, ${ }^{\mathrm{e}}$

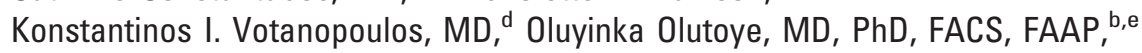 \\ Karen W. Eldin, MD, ${ }^{c, e}$ and Ralph D. Feigin, MD, FAAP, ${ }^{a, e}$ Houston, Tex
}

\section{Clinical Summary}

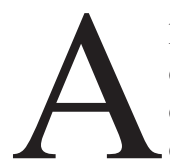

healthy 16-year-old African American girl, who was a competitive track athlete, presented to her primary care clinic with a new, intermittent, sharp, left lateral chest pain with Valsalva maneuvers, aggravated by deep inspiration. There were no associated symptoms such as shortness of breath, coughing, diaphoresis, or abdominal pain.

A chest radiograph was interpreted as showing left pneumothorax, and the patient was sent to the Texas Children's Hospital Emergency Center for further evaluation and management.

A repeat chest radiogram (Figure 1) revealed a large round mass occupying much of the left middle and lower hemithorax, obscuring the left cardiac border. A computed tomographic scan of the chest (Figure 2) revealed an approximately $20-\mathrm{cm}$ multiloculated, cystic mass seated on the left hemidiaphragm, the wall of which appeared to be continuous with the anterior pleura or pericardial sac. The initial serum studies (sodium, potassium, chloride, bicarbonate, blood urea nitrogen, creatinine, glucose, and uric acid) were within normal limits and the patient was admitted with a working diagnosis of germ cell tumor versus foregut duplication cyst.

After preoperative serum human chorionic gonadotropin (HCG) and alpha-fetoprotein (AFP) levels were determined to be within normal limits, a video-assisted thoracic surgery procedure was performed. Operative findings were significant for a cystic lesion involving half of the left hemithorax. The cyst was attached only on the medial and superior margins to the left lobe of the thymus, which was excised en bloc with the mass. The left phrenic nerve was wrapped around the mass and was preserved. After controlled intraoperative decompression, thick, brownish fluid was obtained and sent for culture, cytologic examination, AFP, and HCG. Results of all these studies were normal. The mass was then removed and a pleural drain was left in place. The pathologic findings consisted of a partially drained multiloculated cyst mea-

From the Departments of Pediatrics, ${ }^{a}$ Pediatric Surgery, ${ }^{\mathrm{b}}$ Pathology, ${ }^{\mathrm{c}}$ and Surgery, ${ }^{\mathrm{d}}$ Baylor College of Medicine, and Texas Children's Hospital, ${ }^{\mathrm{e}}$ Houston, Tex.

Received for publication June 5, 2007; accepted for publication July 19, 2007.

Address for reprints: Cathrine Constantacos, MD, One Baylor Plaza, Houston, TX 77030 (E-mail: constant@bcm.tmc.edu).

J Thorac Cardiovasc Surg 2007;134:1373-4

$0022-5223 / \$ 32.00$

Copyright () 2007 by The American Association for Thoracic Surgery

doi:10.1016/j.jtcvs.2007.07.034 suring $22 \times 11 \times 2.5$ $\mathrm{cm}$ containing amorphous, acellular, fibrinous debris with the wall thickness ranging from

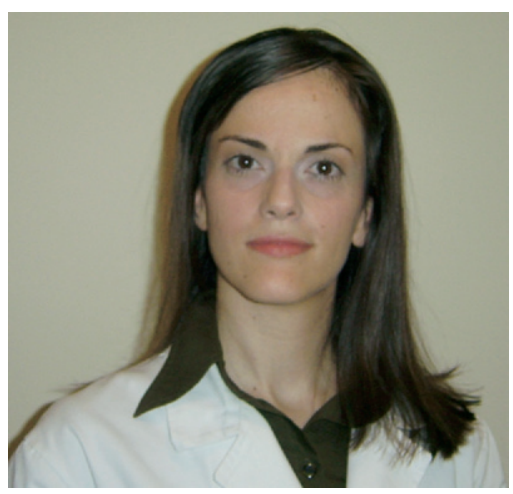

Dr Constantacos 0.3 to $1 \mathrm{~cm}$. The cyst

wall revealed focal calcifications and areas with thymic stroma showing orderly groups of lymphoid cells with scattered Hassall corpuscles, thus confirming the diagnosis of a thymic cyst without evidence of malignancy.

The patient's postoperative course was complicated by a persistent small left pneumothorax that was followed up clinically until resolution. She was discharged in good condition on postoperative day 7 .

\section{Discussion}

Thymic cysts are rare (1\%-3\% of mediastinal cystic lesions), usually benign entities representing remnants of persistent thymopharyngeal duct or cystic degeneration of Hassall corpuscles. ${ }^{1}$ They usually present along the path of descent of the thymic primordia from the angle of the mandible to the anterior mediastinum. ${ }^{2}$ To the best of our knowledge, there have been no published cases of thymic cysts occupying the left hemithorax.

Thymic cysts are usually asymptomatic unless of significant size, thereby causing respiratory or cardiovascular compromise (eg, cough, dyspnea, dysphagia, or congestive heart failure) or chest pain from irritation of the pleurae.

Thymic cysts are classified as either unilocular or multilocular. Unilocular thymic cysts are usually congenital, with thin walls, and contain clear, acellular fluid. On the contrary, multilocular thymic cysts are predominantly acquired, resulting from inflammation or neoplasm; they have thick walls and contain dark-brown fluid. $^{3}$

The differential diagnosis of a hemithoracic cystic lesion includes neoplasms with cystic degeneration (germ cell tumors, Hodgkin disease, thymic carcinoid), postradiation cystic degeneration of the thymus, complex cystic lesions (benign cystic teratoma, thymic cyst, cystic hemangioma, echinococcal cyst), bronchopulmonary foregut duplication cysts, cystic lymphangioma, mesothelial cysts, pancreatic pseudocysts herniating through the diaphragm, thoracic aneurysms, and thoracic duct cysts. ${ }^{4,5}$

Chest radiography, computed tomography, and magnetic resonance imaging can be used to establish the diagnosis of a cystic chest mass and determine its origin and extension. Tumor markers such as sialylated Lewis X-i, carbohydrate antigen 19-9, tissue polypeptide antigen, HCG, carcinoembryonic antigen, AFP, and echinococcal antibodies should be obtained. The decision between a video-assisted thoracic surgery procedure versus thoracotomy 


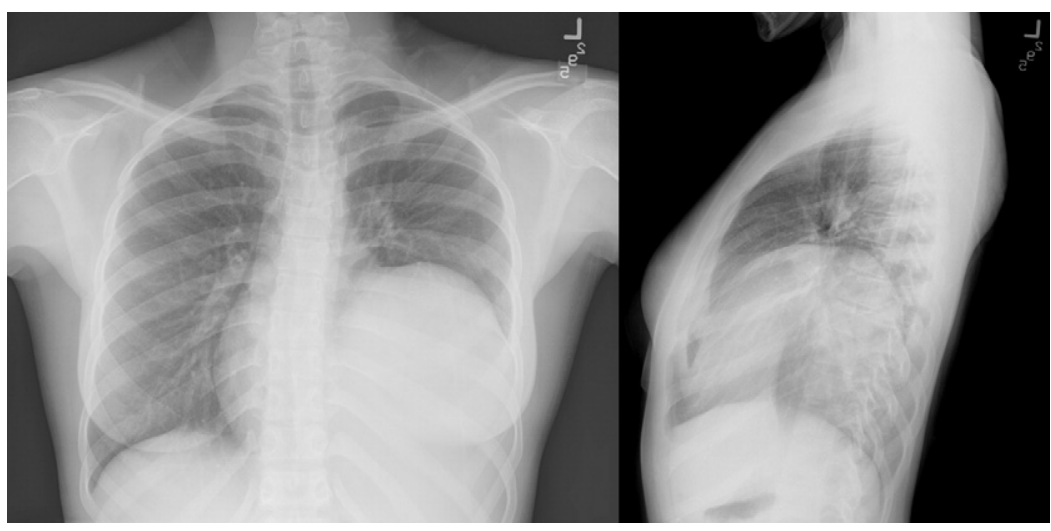

Figure 1. Chest radiographs (posteroanterior and lateral views) at presentation. Note round mass in the left side of the chest occupying approximately half of the left hemithorax.

should be based on cystic size, location, and inability to rule out malignancy. Thymic cysts can coexist with several different malignant tumors. Any concern of concurrent neoplasm (teratoma, Hodgkin disease, thymoma, thymic carcinoma, or neurofibromatosis) should make surgical exploration with complete resection the treatment of choice. Complete surgical excision of an isolated simple thymic cyst is curative.

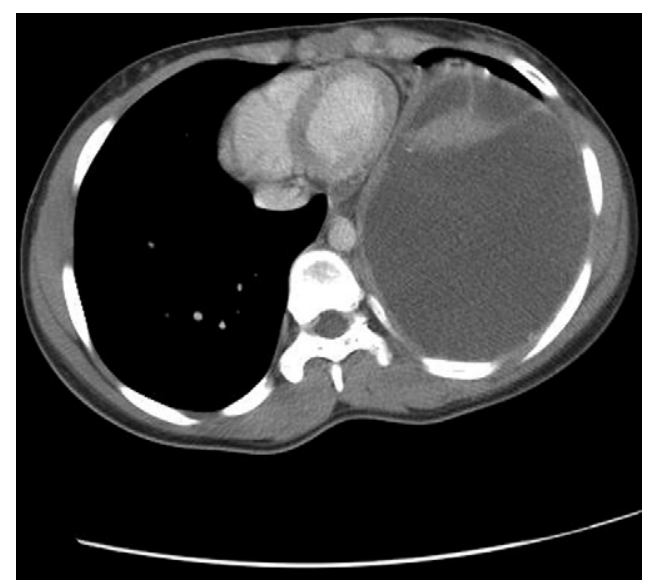

Figure 2. Computed tomographic image of the multiseptated cyst at the mid-level of the heart.
Rare association with diseases like Behcet disease, systemic lupus erythematosus, Sjögren syndrome, human immunodeficiency virus infection, and myasthenia gravis has also been reported.

\section{Conclusions}

The diagnosis of a thymic cyst rarely is considered preoperatively when the lesion is located in the lower portion of the chest. It usually is suspected intraoperatively and confirmed by the pathologist. If the cyst coexists with malignant lesions, treatment must be directed toward the coexisting pathologic condition. The diagnosis of thymic cyst should be entertained in any individual who has compression-related symptoms of thoracic organs and diagnostic imaging findings of a well-defined intrathoracic cystic lesion, regardless of its location within the chest.

\section{References}

1. Speer FD. Thymic cysts. NY Med Coll Flower Hosp Bull. 1938;1: $142-50$.

2. Nguyen Q, DeTar M, Wells W, Crockett, D. Cervical thymic cyst case reports and review of the literature. Laryngoscope. 1996;106: 247-52.

3. Duwe BV, Sterman DH, Musani AI. Tumors of the mediastinum. Chest. 2005;128:2893-909.

4. Takeda S, Miyoshi S, Minami M, Ohta M, Masaoka A, Matsuda H. Clinical spectrum of mediastinal cysts. Chest. 2003;124:125-32.

5. Wick MR. Cystic lesions of the mediastinum. Semin Diagn Pathol. 2005;22:241-53. 